$90 \%$ for gluten) and potent activators of the innate immune system via the toll like receptor 4 (TLR4)-MD2-CD14 complex in cells of the mononuclear phagocyte system (Junker Y et al, J Exp Med 2012), triggering several autoimmune/inflammatory diseases. In contrast, pure gluten that is de-enriched of ATIs shows no stimulatory activity. MRL-Fas(lpr) mice develop progressive and spontaneous glomerular, tubulointerstitial and perivascular kidney disease, arthritis, lymphadenopathy, splenomegaly and circulating autoantibodies in a syndrome that resembles systemic lupus erythematosus (SLE). Here we explored the effect of dietary ATIs on disease severity in MRL-Fas(lpr) mice.

Methods MRL-Fas(lpr) mice were placed either on a glutenfree diet (GFD) or a diet containing 25\% gluten (which contains amounts of ATIs equivalent to the human wheat based diet). We measured serum cytokines, proteinuria, haematuria, hemoglobinuria and histological and immunohistochemical markers of myeloid inflammation (F4/80 and CD68) in different affected organs (kidney, spleen and intestine).

Results Mice on a GFD showed lower levels of serum inflammatory cytokines (IL-6, KC and TNFa) that accompanied lower grade proteinuria, haematuria and hemoglobinuria during the study period. CD68, F4/80 and CD4 positive cells were also higher in the animals that consumed ATIs.

Conclusions Dietary wheat ATIs enhances SLE disease progression, in contrast, a GFD (which is ATI-free) had a protective effect on the development of SLE in MRL-Fas(lpr) mice, confirming the role of ATIs as important nutritional co-stimulant of inflammation in autoimmune diseases.

\section{P69 RISK FACTORS FOR ADVERSE PREGNANCY OUTCOME IN PATIENTS WITH SLE}

${ }^{1}$ Çiğdem Çetin, ${ }^{2}$ Tuğba Saraç-Sivrikoz, ${ }^{2}$ Müge Ateş-Tikiz, ${ }^{1}$ Yasemin Yalçınkaya, ${ }^{1}$ Ahmet Gül, ${ }^{1}$ Lale Öcal, 'Murat İnanç, ${ }^{2}$ ibrahim Kalelioğlu, 'Bahar Artım-Esen. 'Rheumatology Dept., Istanbul Faculty of Medicine, Istanbul University, Istanbul; '2Obstetrics and Gynaecology Dept., Istanbul Faculty of Medicine, Istanbul University, Istanbul, Turkey

\subsection{6/lupus-2020-eurolupus.114}

Objective Lupus pregnancies are considered as high risk because of both disease activation and pregnancy complications. In this study, we determined the risk factors associated with adverse pregnancy outcomes (APO) in pregnant patients with lupus followed up by both Rheumatology and Obstetrics and Gynaecology (O\&G) departments at our university.

Method 136 lupus patients with 168 pregnancies were analyzed. The course of pregnancies and fetal/neonatal prognosis were identified. Unexplained fetal death $\geq 12$ gestational weeks, neonatal death, preeclampsia, eclampsia, preterm birth due to HELLP and/or small for gestational age (SGA) infant were defined as APO. Clinical and laboratory findings, disease activity (SLEDAI-2K) and damage (SLICC/ACR) and conventional risk factors were compared between APO $(+)$ and APO (-) groups.

Results $71 \%$ of pregnancies resulted in live births and APOs occurred in 34\% (table 1). Renal and neuropsychiatric (NP) involvement, thrombocytopenia, antiphospholipid syndrome (APS), lupus anticoagulant and anti-cardiolipin $\operatorname{IgM}$ positivity rates were significantly higher in APO $(+)$ (table 2). Mean SLEDAI-2K during pregnancy and postpartum were higher in APO $(+)(2.2 \pm 3.6$ vs $1.2 \pm 2.04, \mathrm{p}<0.05 ; 4.9 \pm 6.03$ vs $2.7 \pm 5.01, \mathrm{p}=0.02$, respectively). There were significantly more patients with damage in the $\mathrm{APO}(+)$ and their mean damage score was significantly higher $(1.8 \pm 2.1$ vs $0.8 \pm$ $1.3, \mathrm{p}<0.05)$. The frequency of patients with NP, renal, cardiovascular and musculoskeletal damage was significantly higher in the $\mathrm{APO}(+)$ (figure 1).

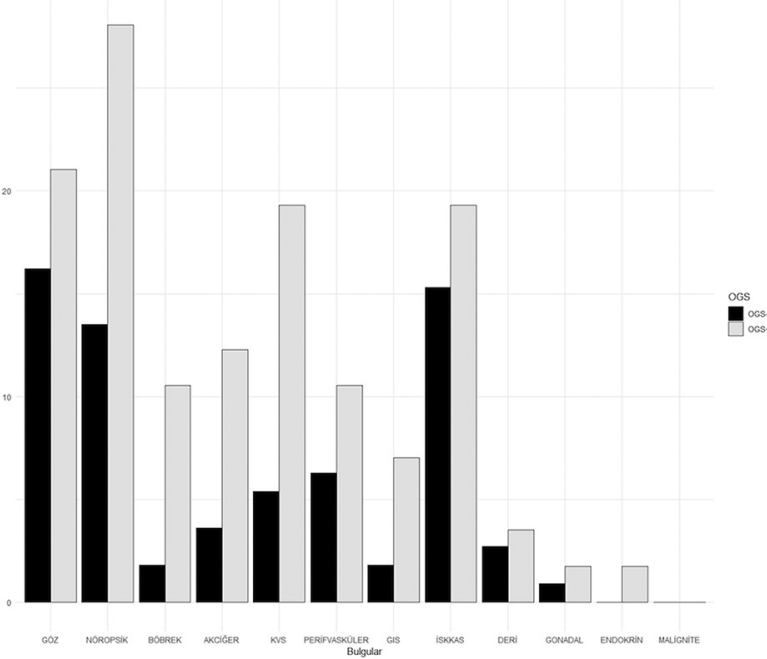

Abstract P69 Figure 1 Distribution of damage according to organs/ systems among APO (+) and APO (-) groups

Abstract P69 Table 1 Pregnancy $(n=168)$, fetal and neonatal outcomes in SLE pregnancy cohort $(n=136)$

\begin{tabular}{ll}
\hline & N (\%) \\
\hline Live birth & $119(70.8)$ \\
$\geq 22$ weeks still born & $11(6.5)$ \\
Neonatal Death & $1(0.6)$ \\
Maternal death & $1(0.6)$ \\
Preterm birth & $37(22)$ \\
SGA birth & $25(14.9)$ \\
Preeclampsia & $14(8.3)$ \\
- <32 weeks & $6(3.6)$ \\
- >32 weeks & $8(4.7)$ \\
HELLP & $5(3)$ \\
Intrauterine growth restriction (IUGR) & $23(13.7)$ \\
- <32 weeks & $13(7.7)$ \\
- >32 weeks & $10(6)$ \\
Pathologic uterine artery Doppler finding & $21(12.5)$ \\
Pathologic umbilical artery Doppler finding & $9(5.4)$ \\
Newborn 1st minute APGAR score <7 & $20(11.9)$ \\
Newborn 5th minute APGAR score <7 & $14(8.3)$ \\
Fetal cardiac disease & $13(7.7)$ \\
-Atrioventricular block & $7(4.1)$ \\
-Sinus bradycardia & $1(0.6)$ \\
-VSD & $2(1.2)$ \\
-VSD+ASD & $1(0.6)$ \\
-Aberrant right subclavian artery anomaly (ARSA) & $1(0.6)$ \\
-Mild hypertrophy of the left ventricle, stenosis in & $1(0.6)$ \\
pulmonary artery index & $13(7.7)$ \\
Fetal/neonatal congenital anomaly or disease & \\
(other than cardiac disease or conduction block & \\
\hline (IUGR: Intrauterine growth restriction, SGA: Small for gestational age) \\
\end{tabular}


Abstract P69 Table 2 Demographic data of APO (+) and APO (-) groups, comparison of conventional risk factors, cumulative clinical, serological and laboratory features

\begin{tabular}{|c|c|c|c|}
\hline & $\begin{array}{l}\text { APO (-) } \\
(n=111)\end{array}$ & $\begin{array}{l}\text { APO (+) } \\
(n=57)\end{array}$ & $\mathrm{p}$ \\
\hline Age & $35.1 \pm 6.7$ & $34.9 \pm 5.9$ & NS \\
\hline Age at conception & $30.6 \pm 5.6$ & $28.9 \pm 4.2$ & NS \\
\hline Disease duration (months) & $141.6 \pm 70$ & $166.9 \pm 87.9$ & $<0.05$ \\
\hline Smoking, $\mathrm{n}(\%)$ & $26(23.4)$ & $5(8.8)$ & $<0.05$ \\
\hline Chronic hypertension, n (\%) & $6(7)$ & $11(19.6)$ & $<0.05$ \\
\hline Gestational diabetes, n (\%) & $3(3.5)$ & $6(10.9)$ & NS \\
\hline Photosensitivity, n (\%) & $86(77.5)$ & $43(75.4)$ & NS \\
\hline Malar rash, $\mathrm{n}(\%)$ & $66(59.5)$ & $38(66.7)$ & NS \\
\hline Discoid rash, n (\%) & $8(7.2)$ & $1(1.8)$ & NS \\
\hline Oral ulcer, n (\%) & $11(9,9)$ & $6(10.5)$ & NS \\
\hline Arthritis, n (\%) & $77(59.4)$ & $42(73.7)$ & NS \\
\hline Serositis, n (\%) & $17(15.3)$ & $13(22.8)$ & NS \\
\hline Renal, n (\%) & $39(35.1)$ & $30(52.6)$ & $<0.05$ \\
\hline Hematologic, n (\%) & $78(70.3)$ & $40(70.2)$ & NS \\
\hline Thrombocytopenia, n (\%) & $37(33.3)$ & $30(52.6)$ & $<0.05$ \\
\hline AlHA, $n(\%)$ & $16(14.4)$ & $14(24.6)$ & NS \\
\hline Neurologic, n (\%) & $7(6.3)$ & $9(15.8)$ & $<0.05$ \\
\hline Anti-dsDNA n (\%) & $63(56.8)$ & $35(61.4)$ & NS \\
\hline Anti-Ro n (\%) & $43(37.7)$ & $21(36.8)$ & NS \\
\hline Anti-cardiolipin IgG, n(\%) & $28(25.2)$ & $18(32.1)$ & NS \\
\hline Anti-cardiolipin IgM, n (\%) & $18(16.2)$ & $18(32.1)$ & $<0.05$ \\
\hline Lupus anticoagulant, n (\%) & $26(23.4)$ & $28(49.1)$ & $<0.001$ \\
\hline Antiphospholipid syndrome, n (\%) & $28(25.2)$ & $30(52.6)$ & $<0.001$ \\
\hline
\end{tabular}

Conclusion Although an important proportion of SLE pregnancies result in live birth, active disease, especially renal and NP involvement, and damage increase the risk of complications. Furthermore, the presence of APS or antiphospholipid antibody positivity are important risk factors for APO. In conclusion, patients, especially with damage, should be pre-counselled to be made aware of the risks. Pregnancy should be allowed after disease activity is controlled and a close monitoring with O\&G clinics is essential.

\section{P70 INCREASED WORK LOSS DURING PREGNANCY IN WOMEN WITH SYSTEMIC LUPUS ERYTHEMATOSUS COMPARED TO MATCHED HEALTHY CONTROLS}

\footnotetext{
1,2Birgit Blomjous, ${ }^{1,3}$ Marieke ter Wee, ${ }^{2}$ Carolien Abheiden, ${ }^{1}$ Alexandre Voskuyl, ${ }^{2}$ Johanna de Vries, 'Irene Bultink. 'Dept. of Rheumatology, Amsterdam Rheumatology and Immunology Center, Amsterdam Infection and Immunity, Amsterdam UMC, Vrije Universiteit Amsterdam, Amsterdam; ${ }^{2}$ Dept. of Obstetrics and Gynecology, Amsterdam Reproduction and Development, Amsterdam UMC, Vrije Universiteit Amsterdam, Amsterdam; ${ }^{3}$ Dept. of Epidemiology and Biostatistics, Amsterdam Public Health, Amsterdam UMC, Vrije Universiteit Amsterdam, Amsterdam, The Netherlands
}

10.1136/lupus-2020-eurolupus. 115

Background Women with Systemic Lupus Erythematosus (SLE) might be more vulnerable to reduce or stop working during pregnancy because of the increased risk of pregnancy complications compared to the general population. Therefore, we aim to assess differences in work participation during pregnancy and thereafter between pregnant women with SLE and matched controls.

Methods A case-control study on employment was performed in pregnant women with SLE and matched controls. Matching criteria were age, year of delivery, and number of living infants. Employment was defined as having $\geq 8$ hours/ week of paid work before conception. Interruption or reduction of work for $>1$ week during pregnancy, complete cessation of work for $>1$ week until delivery, and the time in weeks to return to work after maternity leave were assessed.

Results A total of 42 women were included (21 SLE patients, 21 matched controls). Mean SELENA-SLEDAI before pregnancy in SLE patients was 2.6 (SD 2.3). Interruption of work for $>1$ week and/or completely stop working during pregnancy occurred in more women with SLE compared to matched controls $(\mathrm{OR}=9.0,95 \%$ CI [1.1-71.0], $\mathrm{p}=0.04)$ and the duration of sick leave was longer $(p=0.004)$. After delivery, no difference in return to work after maternity leave was found between women with SLE and controls $(\mathrm{OR}=1.0,95 \%$ CI [0.25-4.0], p=1.0).

Conclusion Pregnant women with SLE more frequently stopped working compared to matched controls. These findings warrant improved counseling of these women as well as extra attention of healthcare providers, including company doctors.

\section{P71 SYSTEMIC LUPUS ERYTHEMATOSUS AND PREGNANCY - 10 YEARS' EXPERIENCE FROM A PORTUGUESE TERTIARY CENTER}

${ }^{1}$ António Braga, 'Jorge Braga, ${ }^{2}$ Carlos Vasconcelos. 'Obstetrics Dept., Centro Materno Infantil do Norte, Centro Hospitalar Universitario do Porto, Oporto; ${ }^{2}$ Clinical Immunology Unit, Centro Hospitalar Universitario do Porto, Oporto, Portugal

\subsection{6/lupus-2020-eurolupus.116}

Background Systemic Lupus Erythematosus (SLE) is an autoimmune disease that affects women in childbearing age, so its association with pregnancy is not a rare event. With the analysis of our cohort over the past 10 years, we seek to clarify the clinical characteristics, evolution, impact of the treatment and evaluation of the outcomes of those pregnancies in our institution.

Methods Retrospective descriptive study of all SLE pregnancies supervised in the Autoimmune Disorders and Pregnancy Consultation Unit of our center, from January 2009 to October 2019.

Results 120 pregnancies were included in 91 patients with a mean age of 32.0 years. $36.0 \%$ were primiparous and $26.8 \%$ had history of miscarriage. In $31.2 \%$ of cases, SLE was associated with other autoimmune diseases and in $14.1 \%$ it was associated with anti-phospholipid antibody syndrome. $11.7 \%$ of patients had history of renal involvement. $95 \%$ of all pregnant women continued the previous medication during pregnancy.

Hydroxychloroquine was the most commonly used drug (58.3\% of cases). $14.2 \%$ had clinical or analytical worsening during the course of pregnancy and $11.9 \%$ at postpartum and all of those cases were controlled with adjustment of the medication. $17.0 \%$ of cases had a preterm delivery, of which 